\title{
Mind the Gap! Tailoring Sol-gel Ceramic Mesoporous Coatings on Labile Metal-organic Frameworks through Kinetic Control.
}

Elisa Bindini ${ }^{1}$, Tanja Lüdtke ${ }^{1}$, Dorleta Otaegui ${ }^{2}$, Marco Möller ${ }^{3}$, Ryma Haddad ${ }^{4}$, Cédric Boissière $^{4}$, Sergio Moya ${ }^{1 *}$

${ }^{1}$ Soft Matter Nanotechnology Group,

CIC biomaGUNE, Basque Research and Technology Alliance (BRTA), Paseo Miramón 182, 20014 San Sebastián, Guipúzcoa, Spain

${ }^{2}$ Mass spectrometry platform, CIC biomaGUNE, Basque Research and Technology Alliance (BRTA), Paseo Miramón 182, 20014 San Sebastián, Guipúzcoa, Spain

${ }^{3}$ Electron microscopy platform,

CIC biomaGUNE, Basque Research and Technology Alliance (BRTA), Paseo Miramón 182, 20014 San Sebastián, Guipúzcoa, Spain

4 Laboratoire de Chimie de la Matière Condensée de Paris, Sorbonne University, Place Jussieu 4, 75005 Paris, France

* Corresponding author E-mail: smoya@cicbiomagune.es

ABSTRACT: Surface engineering of metal-organic frameworks (MOFs) with a mesoporous silica coating can improve MOF mechanical properties and provide an easy way to decorate MOF nanoparticles with organic or biological molecules though silane chemistry or electrostatic interactions, while retaining open access to MOF porosity. Silica coating would be highly beneficial for employing MOFs in a wide range of applications such as catalysis or drug delivery. However, obtaining a stable, controlled core-shell structure using MOF nanoparticles as seed is challenging because of their intrinsic chemically labile nature. Here we analyze the factors that destabilize the core of the Zeolitic Imidazolate Framework-8 (ZIF8) MOFs during the sol-gel deposition of a mesoporous silica shell causing a partial or total etching of the MOF material. Silicates in solution are found to scavenge $\mathrm{Zn}^{2+}$ ions removing them from the ZIF structure and causing a partial or complete dissolution of the ZIF seed. By carefully tuning the silicate concentration in solution simultaneous control can be obtained over both the ZIF-8 dissolution and the silica condensation kinetics, resulting in the growth of a uniform mesoporous silica shell while preserving the integrity of ZIF-8. The core-shell nanoparticles obtained show a compact core shell structure with no gap between the MOF core and the silica shell, even after calcination, while the crystalline ZIF-8 structure is retained. Overall, a general synthetic approach is presented for producing nanocomposite 
core-shell materials which can be applied to other MOF labile seed to design new hierarchical materials.

\section{Introduction}

Metal-organic frameworks (MOFs) are a class of hybrid coordination polymers where metal cations or clusters are connected through organic linkers in 3D porous structures. These crystalline materials are highly porous, and display a high surface area and tunable pore size, which make them attractive for a wide range of applications including catalysis ${ }^{1,2}$, gas separation and storage ${ }^{3-5}$, sensing ${ }^{6}$, photonics $^{7}$ and nanomedicine ${ }^{8,9}$. From the mid-1990s a large variety of cations/clusters and linkers have been employed in the synthesis of MOFs resulting in thousands of MOF materials with different crystalline structures, porosity, pore hydrophobicity and size. MOF functionality and the hydrophobicity of pore cavity are usually defined by the nature of the linker ${ }^{10}$. A frequent strategy explored for tuning MOF functionality has been the use of MOFs in composites with other materials such as metal nanoparticles $^{11-14}$, graphene ${ }^{15}$, silica ${ }^{16,17}$ or polymers ${ }^{18,19}$. Also, MOF materials have been hierarchically assembled to obtain core-shell structures with unique properties ${ }^{20}$.

The composite approach is certainly very interesting for the design of materials with tailored properties, but it is often the case that MOF stability is compromised by the interaction with other components in the composite or during composite fabrication, especially if it involves molecules or environmental conditions that can affect coordination between linkers and ions. In fact, MOFs are very well known for their thermal stability up to relatively high temperatures, but their chemistry is labile and the coordination interactions that hold them together can be easily reversed in the appropriate conditions. MOF crystals in solution are very sensitive to $\mathrm{pH}$ changes as linkers usually involve carboxylate or amine groups that protonate/deprotonate according to $\mathrm{pH}$. Besides, the presence of anions competing with the linkers may shift the coordination equilibrium, disassembling the structure. These issues become particularly dramatic for MOF stability when an inorganic coating like silica or a metal is deposited on top of the MOFs, since during the coating MOFs are exposed to different ions and changes in $\mathrm{pH}$. Working with MOFs in solution to synthesize core-shell or composite materials is therefore not straightforward and particular care has to be taken to perfectly control the reaction conditions. This is especially critical with MOF nanocrystals or nanoMOFs, since their high surface area ensures a fast exchange with the solution that may cause a rapid disassembly of the MOF structure. Although the synthesis of nanoMOFs is well-established and they can be produced in large amounts, the vast majority of the chemical stability studies found in the literature are concerned with micron-sized crystals. There may be huge differences in stability between micro- and nanocrystals for the same environmental conditions. Indeed, stability issues can be a significant obstacle to multistep synthesis involving nanoMOFs.

A very important class of MOFs are the so called Zeolitic Imidazolate Frameworks (ZIFs). These MOFs have the same topology as aluminosilicate zeolites, with transition metals such as $\mathrm{Zn}$ and Co tetrahedrally coordinated to imidazolate linkers ${ }^{21}$. Among the ZIF family, ZIF-8 is probably the most studied. $\mathrm{ZIF}-8$ is formed by $\mathrm{Zn}^{2+}$ metal centers and 2-methylimidazole linkers. ZIF-8 displays high thermal stability and a high surface area, making it an excellent candidate for many applications in catalysis, sensing and gas storage. In 2009, Cravillon and 
colleagues reported the first colloidal synthesis ${ }^{22}$ for ZIF-8, paving the way for nanoscale ZIF8 materials. Since then, many studies have been carried out aiming to control the size, homogeneity and shape of nano-ZIF-8, investigating the influence of synthetic conditions such as solvent, molar ratio, reaction time, metal source and additives ${ }^{23}$. Organic molecules, inorganic nanoparticles and biomolecules have been successfully encapsulated in ZIF-8 shells to form multifunctional hybrid particles ${ }^{14,24,25}$. In addition, post-synthesis surface functionalization of ZIF-8 crystals has been reported with $\mathrm{Au}$ nanoparticles ${ }^{26}$, silica ${ }^{17}$ and lipids ${ }^{27}$. In particular, covering ZIF-8 nanoparticles with a mesoporous silica shell has been demonstrated to improve mechanical properties of the MOFs while retaining free access to the ZIF porosity, which is important for applications ranging from catalysis to drug delivery. Moreover, silica offers the possibility of easy and stable surface functionalization with polymers, peptides, antibodies, and fluorescent probes, which, in most cases, is difficult with naked ZIF-8 particles. However, using ZIF-8 as seeds for silica growth is not without its challenges. As discussed above, MOF structures are non-covalent networks and can disassemble rather easily during the growth of a silica shell, leading in best cases to defective yolk-shell ZIF-8@ $\mathrm{SiO}_{2}$ particles with a void in between silica and the MOF, and in the worst cases to the almost complete dissolution of ZIF-8. Examples of this synthetic problem can be found in the literature. Additional synthesis steps are usually needed for reinforcing the structure of particles, or repairing the damaged ZIF-8 structure ${ }^{17}$. As practical applications of a composite hierarchical structure like MOFs/mesoporous silica largely depend on the quality of the particles, we dedicated some work to the determination of the factors that influence the chemical stability and the structural integrity of nano-sized ZIF-8 crystals during the deposition of a mesoporous silica shell. At the same time, we demonstrated how almost perfect core-shell structures can be achieved with high reproducibility, by controlling MOF dissolution kinetics and silica condensation kinetics. Most importantly, we show here an easy method for coating ZIF-8 nanocrystals with a stable mesoporous silica shell without compromising their structure. This silica coating method can serve a variety of applications by providing a useful means to engineer MOF surfaces and to control diffusion into and out of the MOF pores.

\section{RESULTS AND DISCUSSION}

It is well known that ZIF-8 crystals are stable in organic solvents while their solubility in water largely depends on $\mathrm{pH}$. In acidic solutions between $\mathrm{pH} 5$ and $6, \mathrm{Zn}^{2+}$ cations and imidazolates dissociate, causing the MOF structure to fall apart. An often-neglected aspect of MOF stability is the notable influence of ligand competitors. For example, as recently reported, ZIF-8 are not stable in several physiological buffers despite the $\mathrm{pH}$ being higher than $7 .{ }^{28,29}$ This is due to the presence of anions in the buffer such as phosphates, which compete with imidazolates to coordinate the $\mathrm{Zn}^{2+}$ ions, removing the metal centers from the MOF and causing the structure to break up. The same phenomenon may happen in organic solvents during a core-shell synthesis. Depositing a silica shell around a nanosized ZIF-8 can be achieved through a standard sol-gel route, employing silica precursors such as tetraethylorthosilicate (TEOS) in alkaline hydroalcoholic solution, as reported by Li et $\mathrm{al}^{17}$ (Figure 1). Mesoporous silica is obtained in a hydro-alcoholic ZIF-8 solution with silica precursors (typically silicon alkoxides) and cationic surfactants which self-assemble into micelles and interact with the cationic hydrophilic external surface of MOF nanoparticles and 
with the hydrophilic silica precursors. The alkoxide hydrolysis and condensation reactions are catalyzed by a base (Figure 1b), and silica is formed around the micelles. Once the surfactant is removed by thermal treatment, a material with well controlled size, shape and dual micro/mesoporous texture is obtained. ZIF- 8 are stable in moderately alkaline $\mathrm{pH}$ and the solvent conditions employed, however, during our first attempts to grow a silica shell we obtained continuous hollow silica capsules, where the ZIF nanoparticles had been completely dissolved (Figure S1). This is because silicate species form zinc-silicate complexes with $\mathrm{Zn}^{2+}$ in alkaline conditions, disrupting and dissolving the ZIF structure. The formation of such complexes between the zinc (II) cations and silicate monomers and oligomers has been observed by NMR, demonstrating the ability of aqueous silicon to scavenge $\mathrm{Zn}^{2+}{ }^{30}$

a)

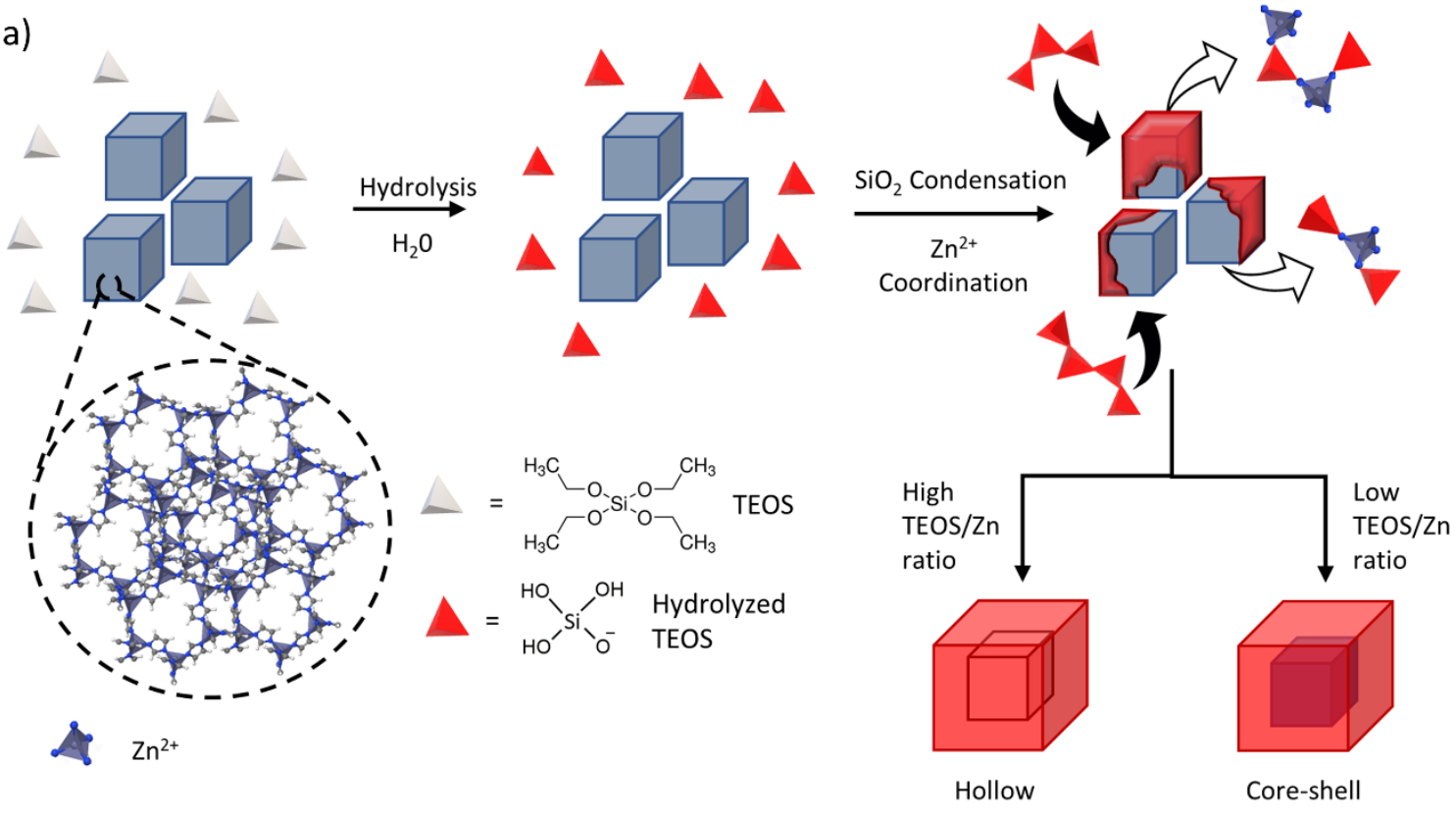

2-methylimidazole

b)

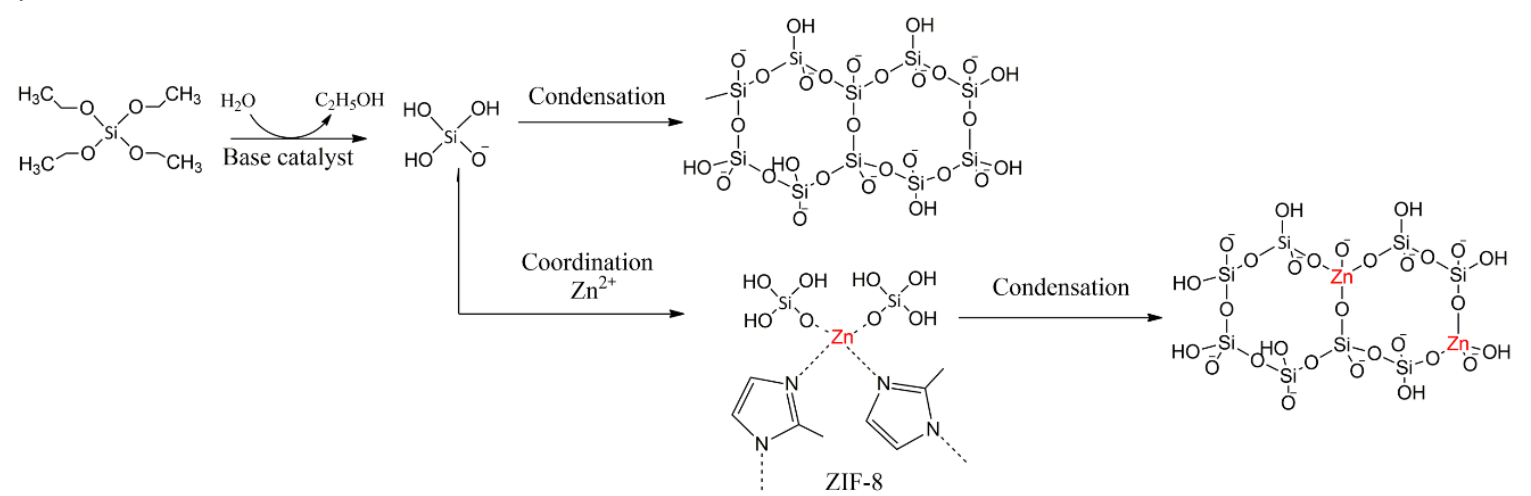

Figure 1: a) Scheme of the formation of a mesoporous silica shell on ZIF-8 nanoparticles, involving silica hydrolysis/condensation equilibria and Zn coordination by silicates. The selected TEOS/Zn ratio will induce either hollow silica capsules doped with $\mathrm{Zn}$ or core-shell particles. b) Main condensation/coordination reactions involved in the mesoporous coating of the ZiF8. The scheme shows a hypothetical encapsulation of $\mathrm{Zn}$ in the mesoporous silica network, following Zn coordination by silicates. The structure of the ZIF-8 has been taken from chemtube3.com.

2.1 Silicate concentration governs ZIF-8 seed dissolution during silica shell growth In the transmission electron microscopy (TEM) micrographs from hollow particles formed after 
condensation of silica on ZIF8, we can recognize that the cavities retain the crystal shape of the MOF particles (Figure S1), meaning that the deposition of the first layer of silica is a fast process on intact ZIF crystals, and only later the $\mathrm{Zn}^{2+}$ is consumed by silicates. This behavior also appears to be visible in some of the TEM images reported by $\mathrm{Li}$ et al ${ }^{17}$, though the micrographs have low contrast. Moreover, some ZIF particles remained inside the silica shell, although slightly etched, to form a yolk-shell structure.

These observations suggest that controlling the concentration of silicate in solution may regulate the ZIF-8 dissolution kinetics, and avoid the ZIF etching during the silica shell growth. To maintain silica hydrolysis and condensation rates almost unaltered, we varied the TEOS/Zn ratio while keeping base concentration and methanol/water ratio constant. Even if TEOS hydrolysis and silica condensation rates are affected by TEOS concentration, with this procedure we avoid the influence of alcohol/water ratio and $\mathrm{pH}$ on the overall kinetics.

During the synthesis of the silica shell, we collected aliquots of the reaction mixture, centrifuged them to separate the particles, and analyzed the supernate by inductively coupled plasma mass spectrometry (ICP-MS) to determine $\mathrm{Zn}^{2+}$ content. At a reaction time of 15 minutes, we observed that the supernate content of $\mathrm{Zn}^{2+}$ was proportional to the TEOS concentration. Over time we observed a decrease in the content of $\mathrm{Zn}^{+2}$, which becomes more significant with higher TEOS concentrations. Since TEOS hydrolysis takes place with an excess of water and catalyst, we can assume that it is a first order reaction. In such a case, the production rate of silicate is proportional to the amount of TEOS introduced into the reactor. This points out that $\mathrm{Zn}^{2+}$ cations are scavenged by silicates (Figure 2). For a TEOS/Zn molar ratio (r) of 55 the $\mathrm{Zn}$ content in the supernate dropped from $1.67 \mu \mathrm{g} / \mathrm{mL}$ to $0.98 \mu \mathrm{g} / \mathrm{mL}$ to 0.65 $\mu \mathrm{g} / \mathrm{mL}$ after 15, 30 and 45 minutes of reaction, respectively. When employing a TEOS/Zn molar ratio of 27 and 15 this decrease was less pronounced but clearly visible. This means that some $\mathrm{Zn}^{2+}$, which was present in solution after 15 minutes of reaction precipitated over time, diminishing the $\mathrm{Zn}^{2+}$ content in the supernate. If we assume that the $\mathrm{Zn}^{2+}$ found in solution was complexed by silicates, we can imagine that when silica condensation took place, a mixed zinc/silicate material was actually formed precipitating on the MOFs. Therefore, the final silica shell should contain $\mathrm{Zn}^{2+}$ previously etched from ZIF substrates, as sketched in Figure 1. In this scenario, high concentrations of TEOS scavenge large quantities of $\mathrm{Zn}^{2+}$ ions in the beginning of the reaction. However, because condensation is fast, $\mathrm{Zn}^{2+}$ quickly reprecipitates along with silica, remaining embedded in the silica shell and causing a sharp drop in the $\mathrm{Zn}^{2+}$ level in solution. 


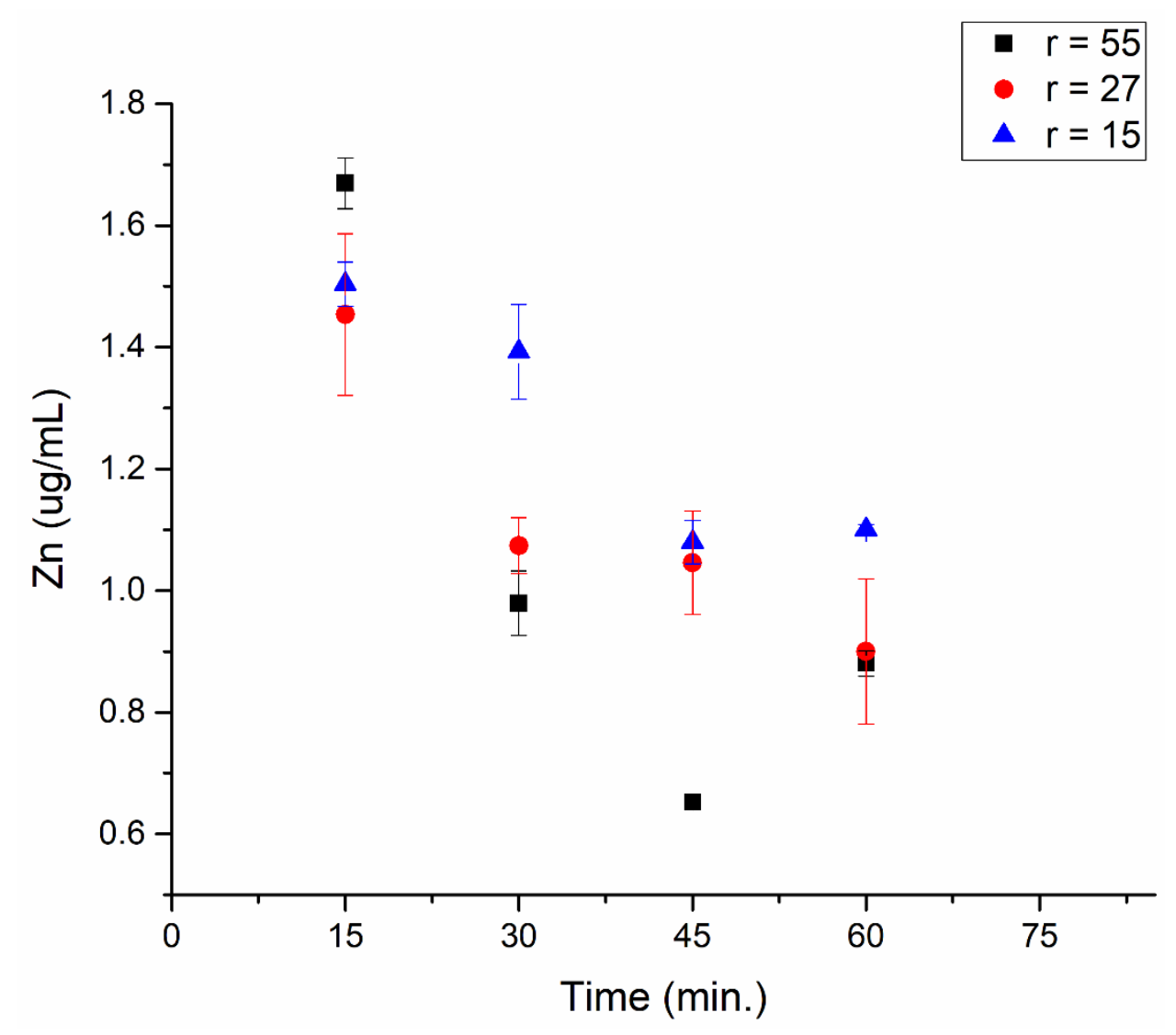

Figure 2: Zn content in solution at different times during the hydrolysis and condensation of silica on top of ZIF-8. Zn content was determined from the supernate by ICP-MS after centrifuging the NPs. Data are reported at different molar ratios ( $r$ ) of TEOS/Zn for the first hour of reaction.

For low TEOS concentrations, fewer $\mathrm{Zn}^{2+}$ ions are scavenged by silicates and $\mathrm{ZIF}-8$ particles are able to retain their shape, as shown by TEM images (Figure 3). Because condensation is slower due to a reduced amount of silicate in solution, the decrease in free $\mathrm{Zn}^{2+}$ concentration due to precipitation over time is less significant (r 27 and 15 in figure 2).

From TEM images we can confirm that with higher concentrations of TEOS $(r=55)$ a silica shell is quickly obtained after $1 \mathrm{~h}$ of reaction but ZIF-8 dissolves, yielding hollow silica capsules (Figure 3a). From energy dispersive X-ray (EDX) analysis, $\mathrm{Zn}^{2+}$ was found embedded in the silica structure, supporting our mechanistic hypothesis (Figure 4a). Moreover, the EDX analysis evidenced the presence of $\mathrm{Zn}$ and $\mathrm{N}$ all over the supporting copper grid, indicating that ZIF-8 crystals disassembled. Decreasing the amount of TEOS to a value of $r=27$ we noticed that silica condensation is slowed down, as is logical, because there are fewer silanols present in solution. After $1 \mathrm{~h}$ of reaction we did not observe a silica shell around the MOFs (Figure 3b, Figure S2). Z-potential data showed that particles were still positively charged (Figure $3 b$ ), while when silica is present around MOFs, a negative surface Z-potential was recorded (Figure 3a). From TEM images we can still see discrete particles after $1 \mathrm{~h}$ of reaction, meaning that the hydrolyzed silicon alkoxide did not complex enough $\mathrm{Zn}$ (II) to remove the ZIF structure. Nevertheless, many holes are visible in the particle's structure, hinting that the MOF crystals were strongly etched during the reaction. Moreover, hydrolyzed silicon precursors condensed to some extent, creating oligomers that nucleated on 
MOF cores, decreasing their surface charge from $+43.8 \mathrm{mV}$ to $+16.4 \mathrm{mV}$. The decrease of zeta potential leads to particle aggregation, precipitating from the suspension.

Further decrease in TEOS/Zn ratio to $\mathrm{r}=15$ yielded almost unmodified ZIF-8 particles after 1 $\mathrm{h}$, with very few visible signs of etching and a Z-potential similar to that of pristine ZIF-8 crystals: $+35.6 \mathrm{mV}$ and $+43.8 \mathrm{mV}$ respectively (Figure $3 \mathrm{c}$ and $3 \mathrm{~d}$ ).

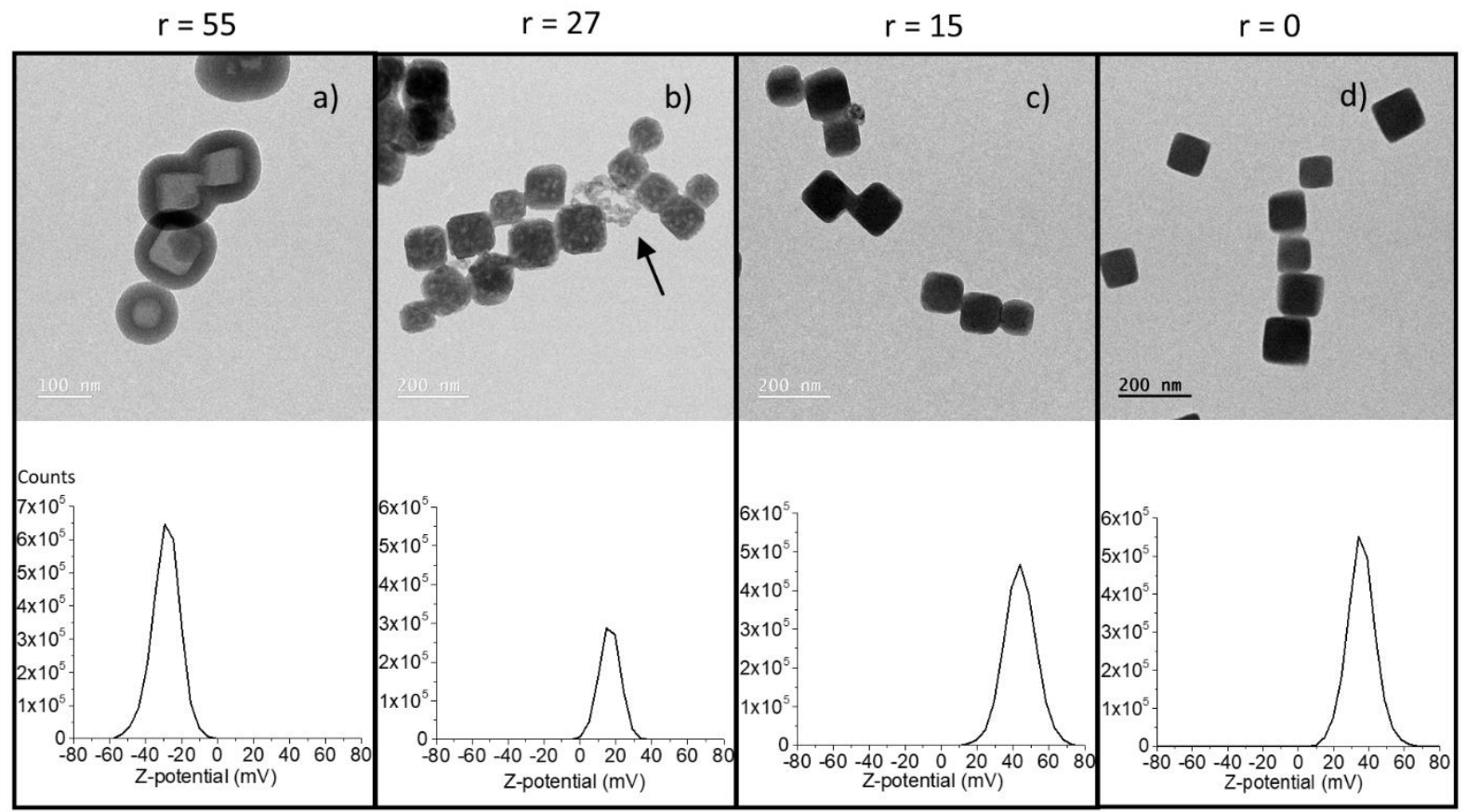

Figure 3: TEM images and Z-potential of ZIF-8@mSiO 2 after $1 \mathrm{~h}$ of reaction at different molar ratio of TEOS/Zn (r). For a) $r=$ 55, b) $r=27$, and c) $r=15$; d) shows data from pristine ZIF-8 particles. The arrow points to structures created by silicate oligomer nucleation.

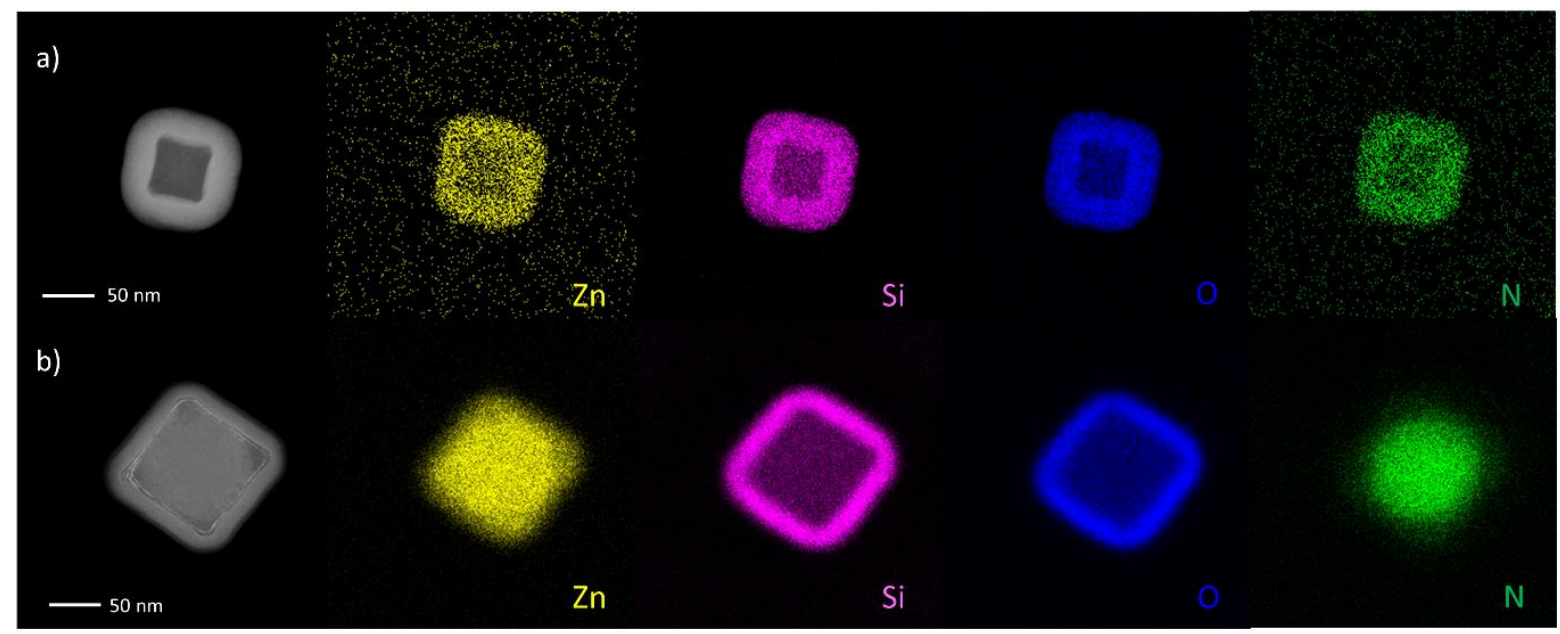

Figure 4: STEM images and corresponding elemental mappings of ZIF-8@mSiO 2 at TEOS/Zn molar ratios of $\boldsymbol{a}$ ) $r=55$ and $\boldsymbol{b}) r$ $=15$.

2.2 Growth of a mesoporous silica shell on ZIF-8 nanoparticles by controlling MOF dissolution kinetics. 
After establishing that by adjusting the proper silicate concentration we could prevent ZIF-8 dissolution during the silica shell formation, we looked for experimental conditions that could optimize the formation of a core-shell structure. Employing a TEOS/Zn ratio $r=15$ and letting the reaction proceed for $3 \mathrm{~h}$ resulted in a nice mesoporous silica shell around the ZIF- 8 cores, which retained their shape with only minimal etching (Figure 5 and Figure S3). The Zpotential of the nanoMOFs became negative, $-28.8 \mathrm{mV}$, proving the presence of silica on the surface while the core-shell structure was confirmed by EDX and X-ray diffraction (XRD) analysis (Figures $4 \mathrm{~b}$ and 5). The $\mathrm{N}_{2}$ adsorption isotherm confirmed a mesoporous structure in the shell with mesopores of $2.3 \mathrm{~nm}$ according to BJH model (Figure $5 \mathrm{f}$ and Figure S5).

In summary, high quantities of free silicates in solution scavenge $\mathrm{Zn}^{2+}$ ions, quickly disrupting the ZIF-8 nanocrystal structure. Later on, silicate-Zn conjugates condense, precipitating a silica shell with $\mathrm{Zn}$ atoms embedded in it. This behavior was clearly observed by EDX studies (Figure 4a) were $\mathrm{Zn}$ and $\mathrm{N}$ atoms, originally from the ZIF crystals, were homogeneously distributed in the silica shell, co-localizing with $\mathrm{Si}$ and $\mathrm{O}$ atoms. It is interesting to notice that this co-localization of $\mathrm{Zn}$ and $\mathrm{Si}$ is also visible in the EDX analysis of $\mathrm{Li}$ and co-workers ${ }^{17}$, where Zn atoms can be spotted in the silica shell. In their case, ZIF-8 etching was only partial and some $\mathrm{Zn}$ could still be seen in the core of the nanoparticles, but there is no doubt that $\mathrm{Zn}$ was also uniformly distributed in the silica shell.

Having understood the dissolution mechanism, we proved that, by decreasing the kinetics of generation of the silicates (by reducing the TEOS/Zn ratio) in solution and by increasing the reaction time to $3 \mathrm{~h}$, it is possible to avoid, or at least to significantly reduce the $\mathrm{Zn}$ scavenging by silicates during the first phase of the synthesis. In this way, core-shell ZIF$8 @ \mathrm{mSiO}_{2}$ nanoparticles are obtained that retain a core of crystalline ZIF-8, as evidenced by XRD spectra (Figure 5g, Figure S3, S4, S6). Corresponding EDX results showed that Zn and $\mathrm{N}$ atoms are only found in the core while $\mathrm{Si}$ and $\mathrm{O}$ are located in the shell (Figure $4 \mathrm{~b}$ ). 

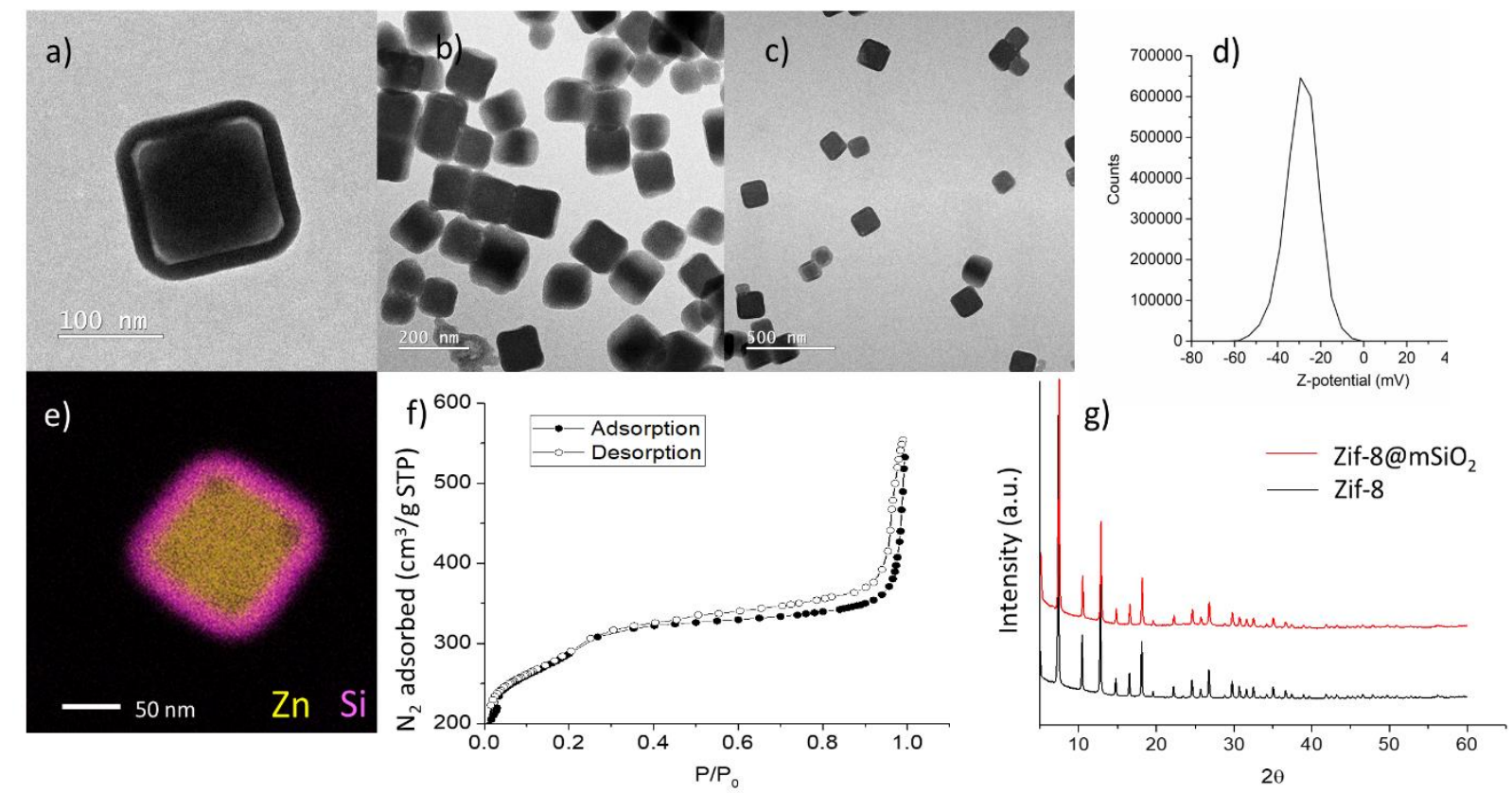

Figure 5: a-c) TEM images d) Z-potential e) EDX elemental mapping f) $N_{2}$ adsorption-desorption isotherm and $g$ ) XRD analysis of ZIF-8 NC@ $\mathrm{mSiO}_{2}$ with a TEOS/Zn molar ratio of 15 after $3 \mathrm{~h}$ of reaction.

The key factor to be successful in this strategy is the balance between a low amount of hydrolyzed silicon alkoxides and a fast silica condensation, which will quickly consume the hydrolyzed monomers and oligomers, thus avoiding their interaction with $\mathrm{Zn}$ atoms. Controlling the amount of precursors can minimize the complexation process and, if silica condensation is fast enough, a silica shell can be obtained without significant etching of the MOFs. Any factor that accelerates silica condensation could in principle favor the shell synthesis, but a fast condensation when performing shell synthesis on nanoparticles also increases the risk of forming aggregates.

The kinetic control approach was proved to be successful and robust with both ZIF-8 nanocubes (NC) (Figure S3) and rhombic dodecahedra (RD) crystals (Figure 6, Figure S4) in obtaining a ZIF-8@ $\mathrm{mSiO}_{2}$ core-shell structures. ZIF-8 signals in XRD were high and clear in every prepared batch (Figure S6), leaving no doubt about the presence of a crystalline ZIF-8 core. The $\mathrm{N}_{2}$ adsorption isotherms confirmed the mesoporous structure of the silica shell and the calculated Brunauer-Emmett-Teller (BET) surface area was $1003.4 \pm 5.7 \mathrm{~m}^{2} \mathrm{~g}^{-1}$ for NC (Figure 5) and $1226.1 \pm 4.6 \mathrm{~m}^{2} \mathrm{~g}^{-1}$ for RD nanoparticles (Figure 6). A closer analysis of isotherms gives a measure of the quality of the final materials after calcination. Indeed, the measure of the height of the hysteresis loop just before the catastrophic desorption at $\mathrm{P} / \mathrm{P}_{0}=0.55$ gives an accurate measure of the mesoporous volume embedded in between the remaining ZIF seed and the silicate shell (it equals the volume of ZIF having been dissolved by silicate species). In our controlled synthesis conditions, the volume of ZIF-8 dissolved are found to be $4.2 \%$ and $1.5 \%$ of the total porous volume of the materials respectively for ZIF-8 $\mathrm{RD} @ \mathrm{SiO}_{2}$ and ZIF-8 NC@ $\mathrm{SiO}_{2}$, while it is more than $10 \%$ for the materials reported in ref 17 for example. We believe that this analysis criteria is a valuable indicator that allows the optimization of the synthetic protocols for producing core-shell materials with a robust 
physical contact in between MOFs and silica shell, and preventing the need for posttreatments to regrow ZIF-8. ${ }^{17}$

Another aspect to take into account to produce an optimal ZIF-8@mesoporous silica NPs is that the gap between the ZIF seed and the mesoporous silica shell may increase upon calcination, as reported previously ${ }^{17}$. We believe that such a behavior could be correlated with the presence of an intermediate thin layer of hydrated zinc silicate in contact with the ZIF particles. In fact, while silica and ZIF contraction upon calcination at $250^{\circ} \mathrm{C}$ should be minimal, zinc silicate could contract a lot more due to water removal, causing the detachment of the silica shell from the ZIF seed. From TGA analysis we could see that ZIF-8@SiO2 NPs lose more mass than pure silica NPs and pristine ZIF-8 NPs upon calcination at $250{ }^{\circ} \mathrm{C}$, confirming our hypothesis (Figure S7). Nevertheless, when observed by TEM after calcination, our particles did not show any remarkable gap between the ZIF core and the mesoporous shell, demonstrating the efficacy of our synthesis methodology to obtain high quality core-shell NPs (Figure S8). If a layer of hydrated Zn silicate is responsible for the gap formation after calcination, our method allows a minimal formation of this intermediate material, limiting the coordination of $\mathrm{Zn}$ by silicates. As a consequence, upon calcination the contraction is negligible, and the particles retain their core-shell structure intact and compact. 

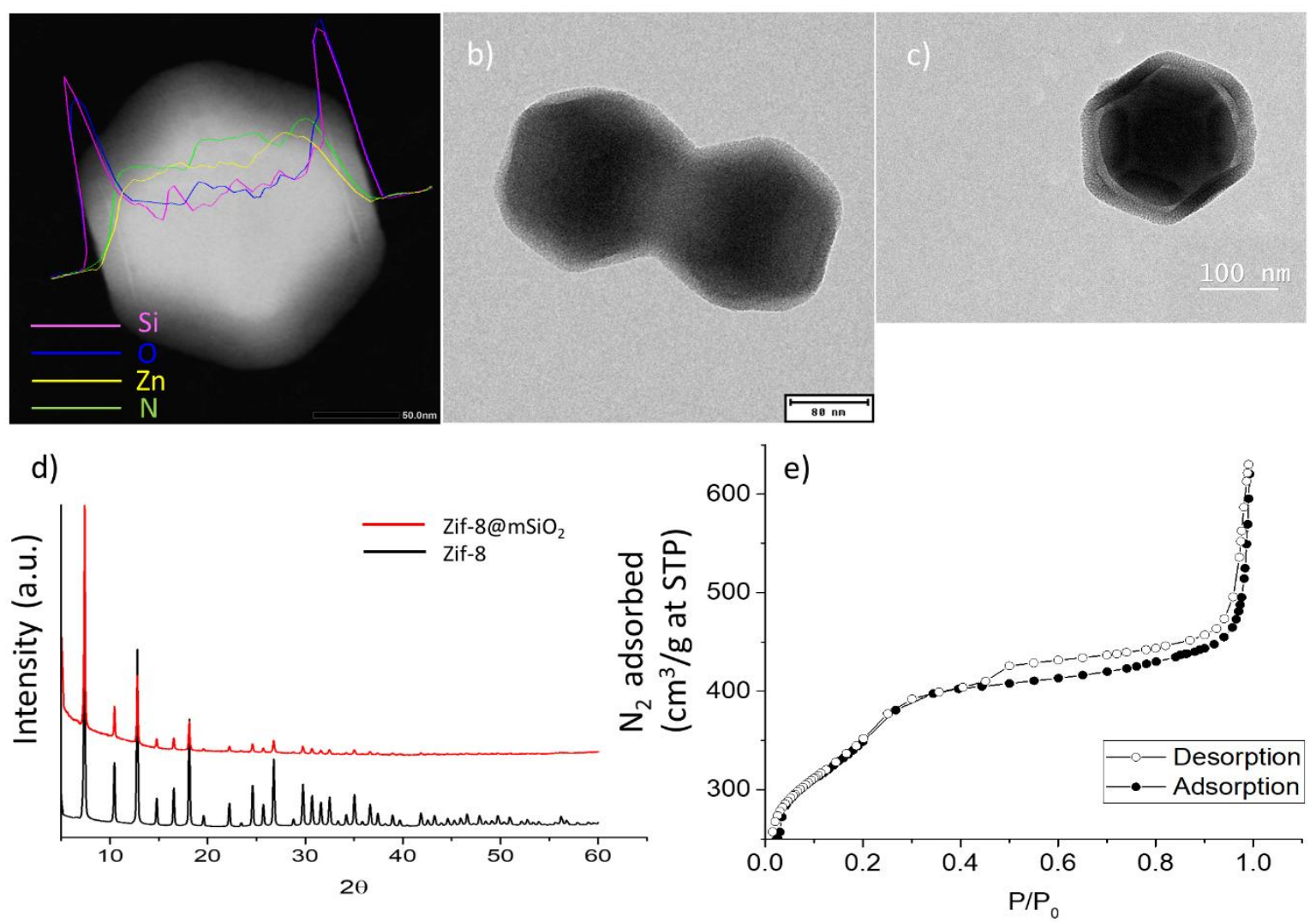

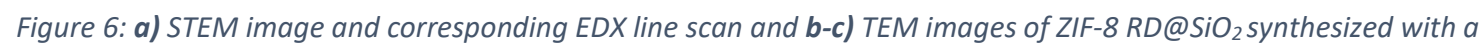
TEOS/Zn ratio $r=15$ d) XRD patterns of ZIF-8 RD nanocrystals and ZIF-8 RD@mSiO ${ }_{2}$ e) $\mathrm{N}_{2}$ adsorption-desorption isotherm of ZIF-8RD@mSiO

\subsection{Effect of 2-methylimidazole as a reaction catalyst.}

The choice of catalyst may impact the hydrolysis/condensation equilibria involved, so we tested the core-shell synthesis with different base catalysts. The results reported until now were obtained in a water/methanol solution, using 2-methylimidazole as the basic catalyst. The reaction can also be performed employing a standard ammonia catalyst with slight variations in concentration to keep a similar $\mathrm{pH}$ (10.1-10.2). Performing the same reaction with ammonia we noticed from ICP data that more $\mathrm{Zn}^{2+}$ was consumed by silicates compared to the 2-mIm synthesis (Figure 7). Moreover, from TEM images we observed that even when a shell was formed, many particles were strongly etched and they changed their shape, looking triangular. Employing $0.0025 \mathrm{M} \mathrm{NH}_{3}$, silica condensation is too slow, and a huge amount of $\mathrm{Zn}^{2+}$ is scavenged by silicates. After $3 \mathrm{~h}$, some mixed aggregates are formed. When increasing the $\mathrm{NH}_{3}$ concentration to $0.025 \mathrm{M}$, silica condensation is faster and after one hour we could see a thick shell around ZIF cores, even if many of them did not retain the cubic shape (Figure 7c). Nevertheless, this fast condensation forms mainly big aggregates and very few isolated core-shell particles. These results evidence a double role of 2-methylimidazole in this reaction. It acts as a base catalyst for TEOS hydrolysis, but being also the ligand of ZIF-8, it contributes to keeping the MOF structure stable by competing with silicate species for $\mathrm{Zn}^{2+}$ coordination, making it a more suitable catalyst than $\mathrm{NH}_{3}$. 

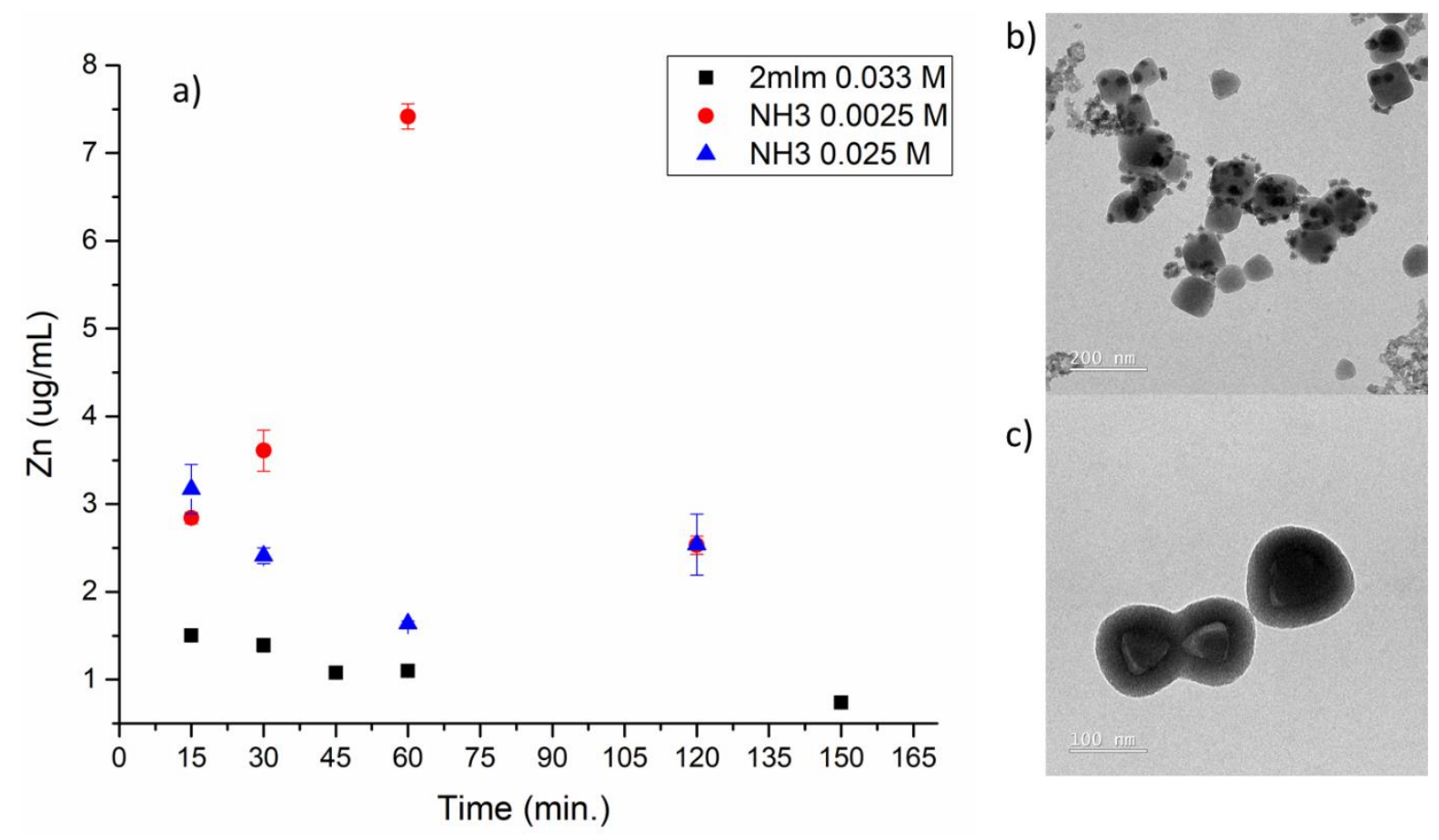

Figure 7: a) Zn content in solution during $\mathrm{SiO}_{2}$ synthesis analyzed by ICP-MS employing different bases and concentrations. Samples were centrifuged and $\mathrm{Zn}$ was determined from the supernatant liquid. TEM images of ZIF-8@mSiO ${ }_{2}$ after 1 h of reaction, employing $\mathrm{NH}_{3}$ at concentrations of b) $0.0025 \mathrm{M}$ and c) $0.025 \mathrm{M}$.

\section{CONCLUSIONS}

We have analyzed the different factors that can lead to the dissolution of ZIF- 8 during the growth of a mesoporous silica shell on a MOF core, and established that the dissolution mechanism is due to the complexation of $\mathrm{Zn}^{2+}$ ions by silicates. This process is non-negligible when a labile MOF core such as ZIF-8 is chosen as seed for mesoporous silica, as it can lead to the complete dissolution of the MOF, and to the precipitation of a mixed silicate/zinc material. We developed a general approach based on kinetic control to prepare core-shell nanocomposites having a crystalline ZIF-8 core and a mesoporous silica shell, by defining proper silica precursor/Zn ratios and reaction time. We could establish that optimal conditions for mesoporous silica formation requires low silicate/Zn ratios and several hours' synthesis. As silicates species are capable of complexing many metals cations, we believe that the reported mechanism of dissolution of ZIF-8 can be extended to almost all MOFs nanoparticles (with reasonably labile MOF core) and our synthesis protocol can be employed to obtain coreshell or hollow metal-silicates nanoparticles with high reproducibility, which will be a valuable tool for generalizing the use of NanoMOFs in composite structures or for developing facile routes for MOFs functionalization. 


\section{Experimental section}

ZIF-8 NC synthesis: $0.27 \mathrm{~mL}$ of a $10 \mathrm{mM}$ solution of CTAB in water were added to $4 \mathrm{~mL}$ of a 1.32 $\mathrm{M}$ solution of 2-methylimidazole in water and left stirring at r.t. for 15 minutes. Then, $4 \mathrm{~mL}$ of a $24 \mathrm{mM}$ solution of $\mathrm{Zn}\left(\mathrm{NO}_{3}\right) \cdot 6 \mathrm{H}_{2} \mathrm{O}$ in water were added and stirred vigorously for 5 minutes. The reaction mixture was left still at r.t. for $3 \mathrm{~h}$. Particles were then collected by centrifugation (15 minutes, $13400 \mathrm{rpm}$ ) and washed with methanol x 3 .

ZIF-8 RD synthesis: $148.8 \mathrm{mg}$ of $\mathrm{Zn}\left(\mathrm{NO}_{3}\right) \cdot 6 \mathrm{H}_{2} \mathrm{O}$ and $164 \mathrm{mg}$ of 2-methylimidazole were dissolved in $10 \mathrm{~mL}$ of methanol. The 2-methylimidazole solution was then added to a nitrate solution under stirring and left stirring at r.t. for $1 \mathrm{~h}$. Particles were collected by centrifugation (15 minutes, $10000 \mathrm{rpm}$ ) and washed with methanol $\mathrm{x} 3$.

ZIF-8@ mSiO$_{2}$ synthesis: $2 \mathrm{mg}$ of ZIF-8 were dispersed in $4.5 \mathrm{~mL}$ of methanol. $30 \mathrm{mg}$ of 2methylimidazole and $40 \mathrm{mg}$ of CTAB were dissolved in $5.5 \mathrm{~mL}$ of water and stirred for 15 minutes. The ZIF-8 suspension was then added to the water solution, sonicated for $1 \mathrm{~min}$ and left stirring for $10 \mathrm{~min}$. TEOS was dissolved in $1 \mathrm{~mL}$ of methanol and added dropwise (see Table 2). The mixture was left stirring at r.t. for $3 \mathrm{~h}$ and the particles were collected by centrifugation and washed with methanol $\mathrm{x} 3$.

\begin{tabular}{|l|l|c|}
\hline & $\mu \mathrm{g} \mathrm{Zn} / \mathrm{mg}$ ZIF-8 & $\mathrm{mmol} \mathrm{Zn/mg} \mathrm{ZIF-8}$ \\
\hline ZIF-8 NC & $280.77 \pm 9.63$ & 0.0043 \\
\hline ZIF-8 RD & $187.01 \pm 3.09$ & 0.0029 \\
\hline \multicolumn{2}{|c|}{ Table 1: Zn content in 1 mg of ZIF-8 crystals, measured by ICP-MS. }
\end{tabular}

\begin{tabular}{|l|c|c|}
\hline & ZIF-8 NC & ZIF-8 RD \\
\hline mmol TEOS/mmol Zn $(r)$ & $\mu$ TEOS & $\mu$ L TEOS \\
\hline 55 & 105 & - \\
\hline 27 & 52.3 & - \\
\hline 15 & 29 & 20 \\
\hline 8 & 14.5 & - \\
\hline
\end{tabular}

Table 2: TEOS quantities used for different synthesis conditions.

ICP-MS: Aliquots of $1 \mathrm{~mL}$ from the reaction mixture were centrifuged to separate particles and supernate. Supernates were left $24 \mathrm{~h}$ at $50{ }^{\circ} \mathrm{C}$ to evaporate solvent, then $50 \mu \mathrm{L}$ of $\mathrm{HF}$ and $500 \mu \mathrm{L}$ of $\mathrm{HNO}_{3} 70 \%$ were added and the samples were left to digest for $72 \mathrm{~h}$. Prior to the measurement, samples were diluted 1:11. Measurements were performed on an iCAPQ ICPMS (Thermo Scientific, Bremen, Germany), with autosampler ASX-520 (Cetac Technologies Inc., NE, USA). Data were analyzed with QtegraTM v2.6 (Themo Scientific, Bremen, Germany).

TEM/EDX: Nanoparticles were imaged by transmission electron microscopy (TEM) operated on a LaB6-TEM of type JEOL JEM-1400PLUS (40 kV-120 kV, HC pole piece) equipped with a GATAN US1000 CCD camera (2k x 2k) and on a FEG-TEM of type JEOL 
JEM-2100F (80 kV-200 kV, UHR pole piece) equipped with STEM (BF \& HAADF) and OXFORD INCA EDX systems, and a TVIPS F216 CMOS camera (2k x 2k).

XRD: X-ray diffraction (XRD) measurements were performed using $\mathrm{Cu} \mathrm{K} \alpha$ radiation in a Bruker D8 Advance diffractometer equipped with a Lynx eye detector.

The specific surface area of the materials was obtained by nitrogen adsorption-desorption isotherm collected at $-196{ }^{\circ} \mathrm{C}$ on a ASAP 2010 from micromeretics The samples were outgassed overnight at $250{ }^{\circ} \mathrm{C}$ prior to the analysis. Specific surface area was calculated applying the Brunauer-Emmet-Teller (BET) method for $\mathrm{N}_{2}$ relative pressure in range of 0.05 $<\mathrm{P} / \mathrm{P} 0<0.20$. Pore size distribution was determined by the Barret-Joyner-Halenda $(\mathrm{BJH})$ method.

\section{Funding}

E.B., T.L. and S.E.M thank the ministry of research and innovation of Spain for support through the PID2020-114356RB-I00 Retos project. This work was performed under the Maria de Maeztu Units of Excellence Program from the Spanish State Research Agency - Grant No. MDM-2017-0720.

We thank Julia Cope, a PhD from CIC biomaGUNE, for carefully revising and editing this manuscript.

\section{References}

1. Lee, J. et al. Metal-organic framework materials as catalysts. Chem. Soc. Rev. 38, 1450 (2009).

2. Rogge, S. M. J. et al. Metal-organic and covalent organic frameworks as single-site catalysts. Chem. Soc. Rev. 46, 3134-3184 (2017).

3. Bae, T.-H. et al. A High-Performance Gas-Separation Membrane Containing Submicrometer-Sized Metal-Organic Framework Crystals. Angewandte Chemie International Edition 49, 9863-9866 (2010).

4. Li, J.-R., Kuppler, R. J. \& Zhou, H.-C. Selective gas adsorption and separation in metalorganic frameworks. Chem Soc Rev 38, 1477-1504 (2009). 
5. Eddaoudi, M. Systematic Design of Pore Size and Functionality in Isoreticular MOFs and Their Application in Methane Storage. Science 295, 469-472 (2002).

6. Stassen, I. et al. An updated roadmap for the integration of metal-organic frameworks with electronic devices and chemical sensors. Chem. Soc. Rev. 46, 3185-3241 (2017).

7. Lustig, W. P. et al. Metal-organic frameworks: functional luminescent and photonic materials for sensing applications. Chem. Soc. Rev. 46, 3242-3285 (2017).

8. Simon-Yarza, T., Mielcarek, A., Couvreur, P. \& Serre, C. Nanoparticles of Metal-Organic Frameworks: On the Road to In Vivo Efficacy in Biomedicine. Adv. Mater. 30, 1707365 (2018).

9. Horcajada, P. et al. Metal-Organic Frameworks as Efficient Materials for Drug Delivery. Angew. Chem. 118, 6120-6124 (2006).

10. Tanabe, K. K. \& Cohen, S. M. Postsynthetic modification of metal-organic frameworks - a progress report. Chem. Soc. Rev. 40, 498-519 (2011).

11. Zhao, M. et al. Metal-organic frameworks as selectivity regulators for hydrogenation reactions. Nature 539, 76-80 (2016).

12. Zhu, Q.-L., Li, J. \& Xu, Q. Immobilizing Metal Nanoparticles to Metal-Organic Frameworks with Size and Location Control for Optimizing Catalytic Performance. $J$. Am. Chem. Soc. 135, 10210-10213 (2013).

13. Carrillo-Carrión, C. et al. Aqueous Stable Gold Nanostar/ZIF-8 Nanocomposites for Light-Triggered Release of Active Cargo Inside Living Cells. Angew. Chem. Int. Ed. 58, 7078-7082 (2019).

14. Chen, L., Peng, Y., Wang, H., Gu, Z. \& Duan, C. Synthesis of Au@ZIF-8 single- or multi-core-shell structures for photocatalysis. Chem. Commun. 50, 8651 (2014).

15. Zheng, Y., Zheng, S., Xue, H. \& Pang, H. Metal-Organic Frameworks/Graphene-Based Materials: Preparations and Applications. Adv. Funct. Mater. 28, 1804950 (2018). 
16. Rieter, W. J., Pott, K. M., Taylor, K. M. L. \& Lin, W. Nanoscale Coordination Polymers for Platinum-Based Anticancer Drug Delivery. J. Am. Chem. Soc. 130, 11584-11585 (2008).

17. Li, Z. \& Zeng, H. C. Armored MOFs: Enforcing Soft Microporous MOF Nanocrystals with Hard Mesoporous Silica. J. Am. Chem. Soc. 136, 5631-5639 (2014).

18. He, S. et al. A generalizable method for the construction of MOF@polymer functional composites through surface-initiated atom transfer radical polymerization. Chem. Sci. 10, 1816-1822 (2019).

19. Kitao, T., Zhang, Y., Kitagawa, S., Wang, B. \& Uemura, T. Hybridization of MOFs and polymers. Chem. Soc. Rev. 46, 3108-3133 (2017).

20. Wang, F. et al. Uncovering two kinetic factors in the controlled growth of topologically distinct core-shell metal-organic frameworks. Chem. Sci. 10, 7755-7761 (2019).

21. Chen, B., Yang, Z., Zhu, Y. \& Xia, Y. Zeolitic imidazolate framework materials: recent progress in synthesis and applications. J. Mater. Chem. A 2, 16811-16831 (2014).

22. Cravillon, J. et al. Rapid Room-Temperature Synthesis and Characterization of Nanocrystals of a Prototypical Zeolitic Imidazolate Framework. Chem. Mater. 21, 1410$1412(2009)$.

23. Troyano, J., Carné-Sánchez, A., Avci, C., Imaz, I. \& Maspoch, D. Colloidal metalorganic framework particles: the pioneering case of ZIF-8. Chem. Soc. Rev. 48, 55345546 (2019).

24. Zheng, G. et al. Shape control in ZIF-8 nanocrystals and metal nanoparticles@ZIF-8 heterostructures. Nanoscale 9, 16645-16651 (2017).

25. Liang, K. et al. Biomimetic mineralization of metal-organic frameworks as protective coatings for biomacromolecules. Nat Commun 6, 7240 (2015). 
26. Li, Z. \& Zeng, H. C. Surface and Bulk Integrations of Single-Layered Au or Ag Nanoparticles onto Designated Crystal Planes $\{110\}$ or $\{100\}$ of ZIF-8. Chem. Mater. 25, 1761-1768 (2013).

27. Wuttke, S. et al. MOF nanoparticles coated by lipid bilayers and their uptake by cancer cells. Chem. Commun. 51, 15752-15755 (2015).

28. Velásquez-Hernández, M. de J. et al. Degradation of ZIF-8 in phosphate buffered saline media. CrystEngComm 21, 4538-4544 (2019).

29. Luzuriaga, M. A. et al. ZIF-8 degrades in cell media, serum, and some — but not all— common laboratory buffers. Supramolecular Chemistry 31, 485-490 (2019).

30. Anseau, M. R., Leung, J. P., Sahai, N. \& Swaddle, T. W. Interactions of Silicate Ions with Zinc(II) and Aluminum(III) in Alkaline Aqueous Solution. Inorg. Chem. 44, 8023-8032 (2005). 